Delft University of Technology

\title{
Concave serrations on broadband trailing edge noise reduction
}

Ragni, Daniele; Avallone, Francesco; van der Velden, Wouter

DOI

10.2514/6.2017-4174

Publication date

2017

Document Version

Accepted author manuscript

Published in

23rd AIAA/CEAS Aeroacoustics Conference

\section{Citation (APA)}

Ragni, D., Avallone, F., \& van der Velden, W. (2017). Concave serrations on broadband trailing edge noise reduction. In 23rd AIAA/CEAS Aeroacoustics Conference: 5-9 June 2017, Denver, Colorado [AIAA 20174174] American Institute of Aeronautics and Astronautics Inc. (AIAA). https://doi.org/10.2514/6.2017-4174

\section{Important note}

To cite this publication, please use the final published version (if applicable).

Please check the document version above.

\section{Copyright}

Other than for strictly personal use, it is not permitted to download, forward or distribute the text or part of it, without the consent of the author(s) and/or copyright holder(s), unless the work is under an open content license such as Creative Commons.

\section{Takedown policy}

Please contact us and provide details if you believe this document breaches copyrights.

We will remove access to the work immediately and investigate your claim. 
This is an Accepted Manuscript of an article published in: 23rd AIAA/CEAS Aeroacoustics Conference 5-9 June 2017, Denver, Colorado. ISBN: 978-1-62410-504-3

Available online: https://arc.aiaa.org/doi/10.2514/6.2017-4174

\title{
Concave serrations on broadband trailing-edge noise reduction
}

\author{
D. Ragni, F. Avallone, W.C.P. van der Velden \\ Delft University of Technology, Faculty of Aerospace Engineering, 2629HS, Delft, The Netherlands \\ ${ }^{a}$ Corresponding author: F.Avallone@tudelft.nl
}

\begin{abstract}
The far-field noise and flow field of a novel curved trailing-edge serration (i.e. iron-shaped) are investigated. Spectra of the far-field broadband noise, directivity plots and the flow-field over the iron-shaped serration are obtained from numerical computations performed using a compressible Lattice-Boltzmann solver. The new design is compared to a conventional trailing-edge serration with a triangular geometry retrofitted to a NACA 0018 airfoil at zero degree angle of attack. The iron-shaped geometry is found to reduce far-field broadband noise of approximately $2 \mathrm{~dB}$ more than the conventional sawtooth serration for chord-based Strouhal numbers $S t_{c}<15$. It is found that the larger noise reduction achieved by the iron-shaped trailing-edge serration is due to the mitigation of the scattered noise at the root, effect obtained by mitigating the interaction between the two sides of the serration, by delaying toward the tip both the outward and the downward flow motions present at the root.
\end{abstract}

Keywords: trailing-edge serration, turbulent boundary-layer trailing-edge noise, aeroacoustics, noise reduction.

\section{Introduction}

Broadband trailing-edge noise generated by the scattering of a turbulent flow convecting over the trailing edge of an airfoil [1,2] is a relevant contributor to wind-turbine noise [3]. Many passive mitigation strategies have been proposed to reduce this source of noise. Acoustic measurements, carried out both in wind tunnels and in-field, reported that sawtooth trailing-edge serrations offer the most effective noise reduction per simplicity of design [411]. Many analytical approaches have been proposed [13-17] to predict trailing-edge noise in presence of sawtooth trailing-edge serrations. When compared to experimental results (e.g. $[4,7,10,11])$, the current triangular designs are not able to match the predicted noise reduction intensity. Recently, Lyu et al. [14,17] developed a new semi-analytical model applying Amiet's trailing-edge noise theory [20] to sawtooth trailing edges. Results showed that the predicted noise reduction are closer to the one experimentally measured. Lyu et al. [14,17] detected two non-dimensional parameters that substantially affect noise reduction: $k_{1} \times 2 h$ and $l_{z p}(f) / \lambda$, where $k_{1}$ is the wavenumber in the chordwise direction and $l_{z p}(f)$ is the spanwise correlation-length of the surface pressure fluctuations. The studies of Lyu et al. concluded that far-field noise can be reduced when both $k_{1} \times 2 h$ and $l_{z p}(f) / \lambda$ are much larger than unity.

More recent investigations on the actual flow fields (e.g. $[7,10,21,22])$ have shown that the flow past serrated airfoils is strongly three dimensional, with vortical structures developing along the edges of the serrations. Flow measurements [7,10,24] and computations $[22,25]$ showed that, even at small angles of attack, the turbulent flow tends to seep into the empty space in between serrations. This flow distortion is found to reduce the effective angle seen by the turbulent flow convecting over the edge of the serrations thus reducing the effectiveness of the serrations in mitigating far-field noise [21]. 
Aiming at reducing the broadband far-field noise even further, several variations of the serration geometry were proposed and tested, e.g. brushed [26], sinusoidal [15], slitted [27,28] and even randomly-shaped trailing edges [29]. More recently, it was shown that broadband far-field noise can be reduced, with respect to conventional serrations, by filling the empty space in between serrations with combs or slits [3]. Based on previous experimental observations [7,10], curved trailing-edge serrations may reduce the far field noise by mitigating the negative effect due to the outward and downward flow motions at the root.

In this manuscript, curved trailing-edge serrations are investigated with the commercial Lattice-Boltzmann Method (LBM) solver Exa PowerFLOW. The iron-shaped serrations are compared to more conventional sawtooth trailing-edge serrations with same length $(2 h)$ and wavelength $(\lambda)$. Aim of this paper is to investigate the physical reasons behind the larger noise reduction achieved by the iron-shaped serrations with respect to the conventional sawtooth serrations. In the following study, the serrations are retrofitted to a NACA 0018 airfoil placed at zero angle of attack, similarly as in the reference experiments $[7,27]$.

\section{Computational test-case}

The commercial software package Exa PowerFLOW 5.3b is used to solve the discrete Lattice-Boltzmann equations for a finite number of directions. For a detailed description of the equations used for the source field computations the reader can refer to Succi [33].

The discretization used for this particular application consisted of 19 discrete velocities in three dimensions (known as the D3Q19 model) involving a third-order truncation of the Chapman-Enskog expansion to retrieve the Navier Stokes equations. For three dimensional computations of low Mach number ideal gas flows, this was found to accurately reproduce the flow field [33]. The distribution of particles was solved using the kinetic equations on a Cartesian mesh, with the conventional Bhatnagar-Gross-Krook (BGK) collision term operator [35]. A Very Large Eddy Simulation (VLES) was implemented as viscosity model to locally adjust the numerical viscosity of the scheme [36]. The model consists of a two-equations $k-\varepsilon$ Renormalization Group (RNG) modified to incorporate a swirl based correction that reduces the modeled turbulence in presence of large vortical structures, required for stability of the code. A turbulent wall-model was used to resolve the near-wall region [37].

Due to the fact that the LBM is inherently compressible and it provides a time-dependent solution, the sound pressure field was extracted directly from the computation domain. Sufficient accuracy is obtained when considering at least 16 cells per wavelength for the LBM [38]. The obtained far-field noise was further compared with noise estimated by using an acoustic analogy. For this purpose, the Ffowcs-Williams and Hawkings (FWH) [39] equation was employed. To further detect the location of the noise sources in the near field, the dynamics of co-rotating flow structures was investigated [44].

Computations were performed on a NACA 0018 airfoil with chord length $c=200 \mathrm{~mm}$ and $80 \mathrm{~mm}$ span (Figure 1). The simulation domain size was $12 \mathrm{c}$ in both the streamwise and the wall-normal directions. The outer $2 c$ were modeled as an anechoic outer-layer to damp down acoustic reflections. In spanwise direction, cyclic boundary-conditions were used. A variable mesh resolution in the computational field was employed. Ten refinement regions allowed the first cell to be placed in the viscous sub-layer at $3.9 \times 10^{-4} \mathrm{C}$ above the trailing-edge location, corresponding to $y^{+}=3$. In total, around 150 million voxels were used for the discretization of the domain. Data sampling started after reaching a steady standard deviation of the lift and drag coefficient in the solution (approximately 10 full flow-passes over the 
airfoil chord). The Courant-Friedrichs-Lewy (CFL) number is dependent on the wave propagation velocity and on the smallest voxel size in compressible simulations. With the current cell size and a unit CFL number, the resulting time-step was $1.3 \times 10^{-7} \mathrm{~s}$. The wall-pressure probes had the same size of the local voxel (i.e. $3.9 \times 10^{-4} \mathrm{C}$ at the trailing-edge location and $7.8 \times 10^{-4} \mathrm{C}$ along the rest of the airfoil). Flow data were sampled with frequency $f=30 \mathrm{kHz}$ for 20 flow passes. The prescribed methodology was validated in a previous study by van der Velden et al. [34].

The trailing-edge thickness of the airfoil was kept sharp with $t=1 \mathrm{~mm}\left(t / \mathrm{c}=5 \times 10^{-3} \mathrm{c}\right)$ in order to minimize the tonal noise component due to the vortex shedding ( $t / \delta \approx 0.1$ where $\delta$ is the boundary layer thickness discussed below) [46]. The free-stream velocity was $U_{\infty}=20 \mathrm{~m} / \mathrm{s}$, and the angle of attack was $\alpha=0^{\circ}$. Boundary-layer transition to turbulence was forced at $x / c=0.2$ by means of a serrated strip of height $3 \times 10^{-3} \mathrm{C}$ and length $1.5 \times 10^{-3} \mathrm{C}$ on both sides of the airfoil [34].

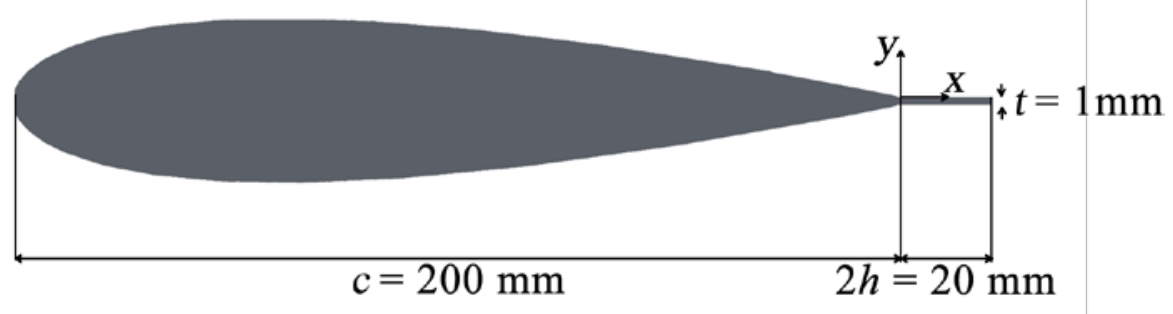

Fig. 1. Sketch of the airfoil (side view).

(a)

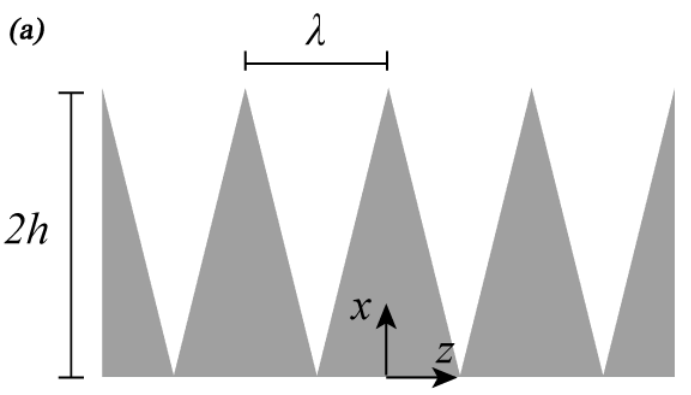

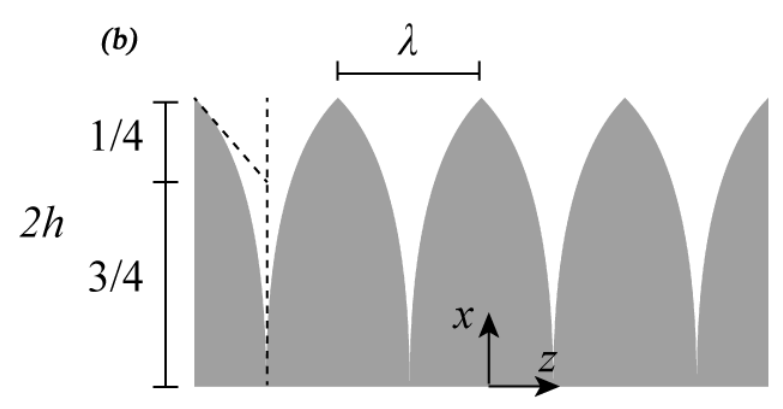

Fig. 2. Sketch of the conventional (a) and iron-shaped (b) trailing-edge serrations.

The airfoil trailing-edge was retrofitted with two serrated trailing-edges: a conventional sawtooth and an iron-shaped geometry (Figure 2). The latter was designed with a spline curve. At the root, it is perpendicular to the baseline trailing-edge while, at the tip, it is tangent to the line obtained as intersection of the tip point with the point at $3 / 4 \times 2 h$ (see dashed line in Figure $2 \mathrm{~b})$. Both serrations had length $2 h / c=0.2(2 h=40 \mathrm{~mm})$ and wavelength $\lambda_{c}=0.1$ $(\lambda=20 \mathrm{~mm})$. The serration length was approximately four times the boundary layer thickness $\delta[7,27]$. The airfoil shape and the geometry of the sawtooth trailing-edge were chosen as in the experiments performed by Arce León et al. [7,27] used as reference throughout the entire manuscript.

The adopted Cartesian coordinate system (Figure 1 and Figure 2) for each configuration is defined as follows: the origin is chosen at the location of the baseline airfoil trailing-edge (i.e., the airfoil with the straight trailing-edge); the $z$-axis coincides with the airfoil trailing-edge; the $x$-axis is aligned with the chord of the airfoil (i.e., aligned with the serration surface); and the $y$-coordinate is perpendicular to the surface of the serrations. In presence of 
serrations, the origin coincides with the projection along the chord direction of a serration tip on the trailing edge of the baseline model (Figure 2).
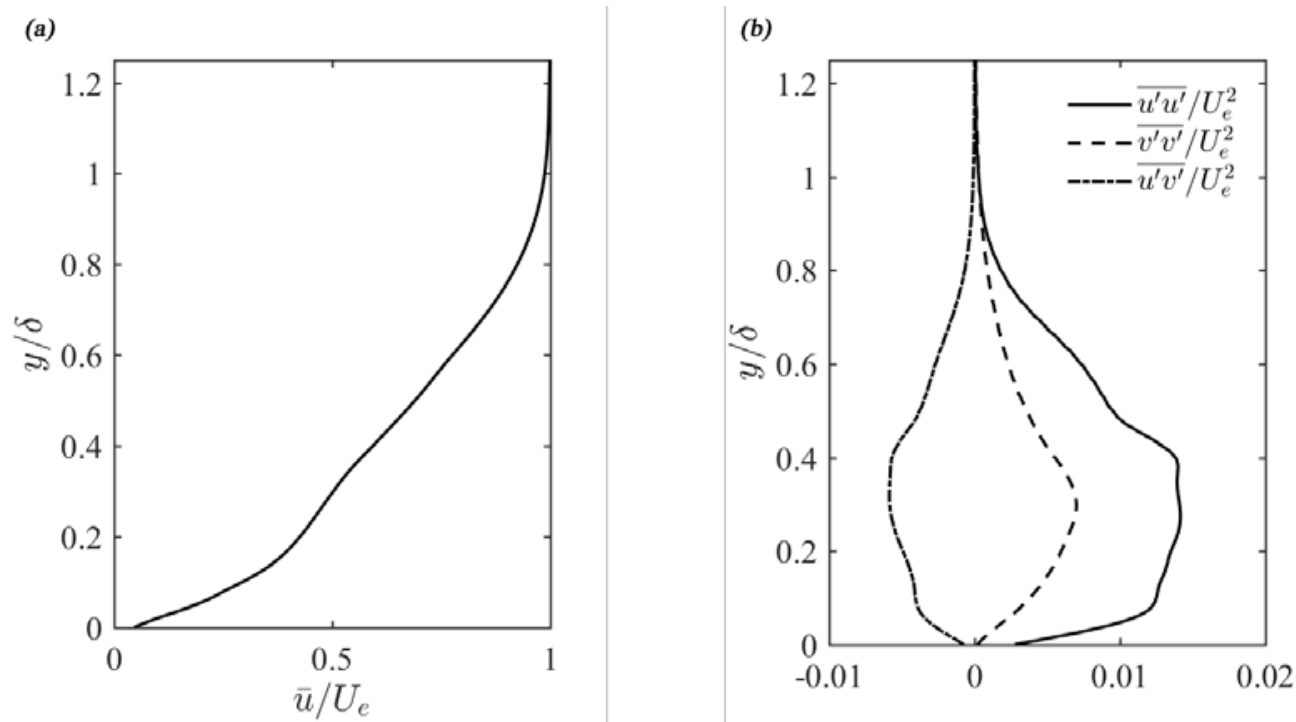

Fig. 3. (a) Mean streamwise velocity component and (b) time-averaged turbulent statistics for the baseline airfoil at $x / c=0 . U_{e}$ is the local edge velocity.

Boundary-layer profiles of the mean streamwise velocity component and time-averaged turbulent statistics [47] were inferred from the computed velocities. They are reported in Figure 3 and summarized in Table I. More in detail, $\delta_{95}=7.9 \mathrm{~mm}$ and $\delta=9.5 \mathrm{~mm}$ are the boundary-layer thickness parameters defined by the $y$-location corresponding to $95 \%$ and $99 \%$ of the local edge velocity $\left(U_{e}\right)$, respectively. $\delta^{*}=3.3 \mathrm{~mm}$ and $\theta=1.5 \mathrm{~mm}$ are the displacement and momentum thickness, respectively. This results in a shape factor $H=\delta^{*} / \theta$ of approximately 2.2. $\delta, \delta^{*}$ and $\theta$ obtained from the computations are similar to the ones reported by Arce León et al. [7] thus allowing the comparison. Table I also lists the corresponding Reynolds numbers for the length scales addressed above.

Table I. Boundary layer characteristics at $x / c=0$

\begin{tabular}{ccc}
\hline \hline Parameter & & Quantity \\
\hline Free-stream velocity & $U_{\infty}$ & $20 \mathrm{~m} / \mathrm{s}$ \\
Edge velocity & $U_{e}$ & $18.75 \mathrm{~m} / \mathrm{s}$ \\
Displacement thickness & $\delta^{*}$ & $3.3 \mathrm{~mm}$ \\
Momentum thickness & $\theta$ & $1.5 \mathrm{~mm}$ \\
Boundary layer thickness & $\delta$ & $9.5 \mathrm{~mm}$ \\
& $\delta_{95}$ & $7.9 \mathrm{~mm}$ \\
& $R e_{c}$ & 280,000 \\
Reynolds number & $R e_{\delta^{*}}$ & 4,600 \\
& $R e_{\theta}$ & 2,100 \\
Shape factor & $\operatorname{Re}_{\delta}$ & 13,300 \\
\hline \hline
\end{tabular}

Given the flow similarities over the different serrations present in the computational domain and in order to further reduce the uncertainty on the velocity fields, the computed fields were spatially averaged along the spanwise direction at points with the same relative 
spanwise location with respect to the serration root. This procedure reduces the uncertainty by increasing the number of samples available for averaging [22].

\section{Results}

\subsection{Far-field noise}

The acoustic power spectra $\left(\Phi_{a a}\right)$ are obtained from the time series of the pressure fluctuations sampled by a microphone located above the trailing edge of the baseline airfoil $(x / c=0, z / c=0$ and $y / c=10)$. Far-field pressure spectra $\left(\Phi_{m}\right)$ are extracted from the FWH analogy [39], and converted to acoustic far field spectra as described in Section 2. Results presented in Figure 4 are scaled as reported in equation 1:

$$
\Phi_{a a}=\Phi_{m}+20 \log _{10}\left(\frac{R_{m}}{R_{r e f}}\right)+10 \log _{10}\left(\frac{b_{r e f}}{b_{m}}\right)+50 \log _{10}\left(\frac{M_{\infty r e f}}{M_{\infty m}}\right)
$$

(a)

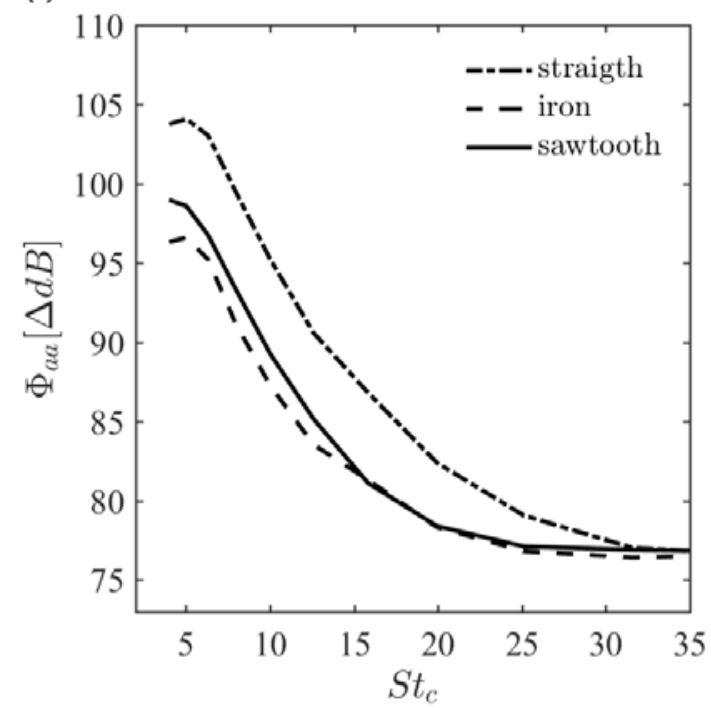

(b)

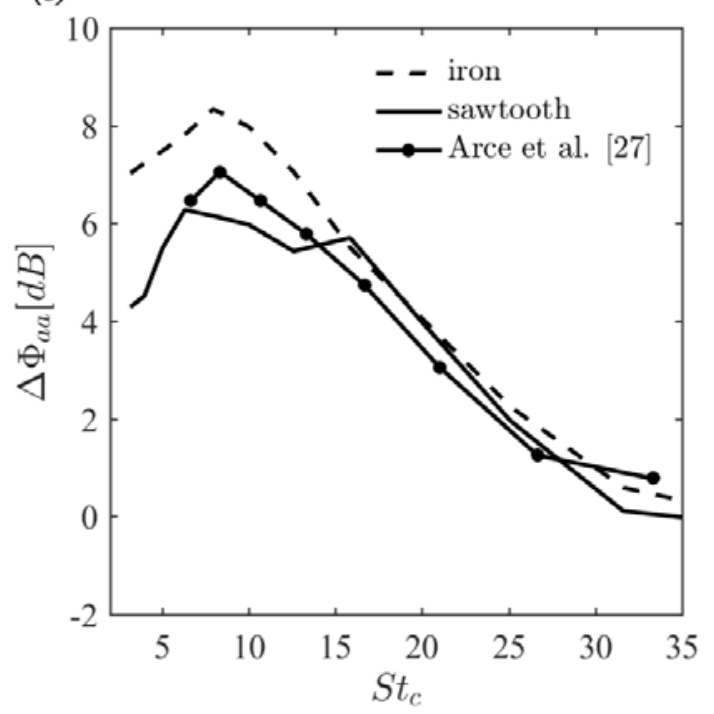

Fig. 4. (a) Power spectra of the far-field pressure fluctuations $\left(\Phi_{a a}\right)$ for the straight, sawtooth and iron-shaped trailing-edge serrations. (b) noise reduction $\left(\Delta \Phi_{a a}\right)$ with respect to the straight trailing-edge noise. Experimental data are taken from Arce et al. [27] at $U_{\infty}=30 \mathrm{~m} / \mathrm{s}$ with the same sawtooth geometry. Computational data are obtained by a microphone located above the trailing edge of the baseline airfoil $(x / c=0, z / c=0$ and $y / c=10)$.

Here, the subscript $m$ indicates the measured value while the subscript ref indicates the reference values. The reference values are: Mach number $M_{r e f}=1$, observer radius $R_{r e f}=1 \mathrm{~m}$ and spanwise length $b_{\text {ref }}=1 \mathrm{~m}$. The adopted scaling is conventionally used in literature for trailing-edge noise studies, where non-compact noise sources are the most relevant contributors $[48,49]$. 
(a)

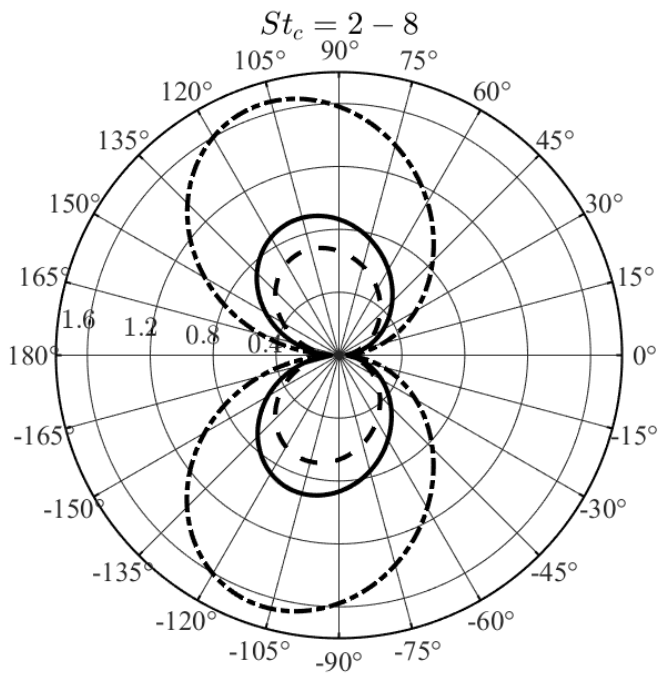

— sawtooth - - iron ----s straight (b)

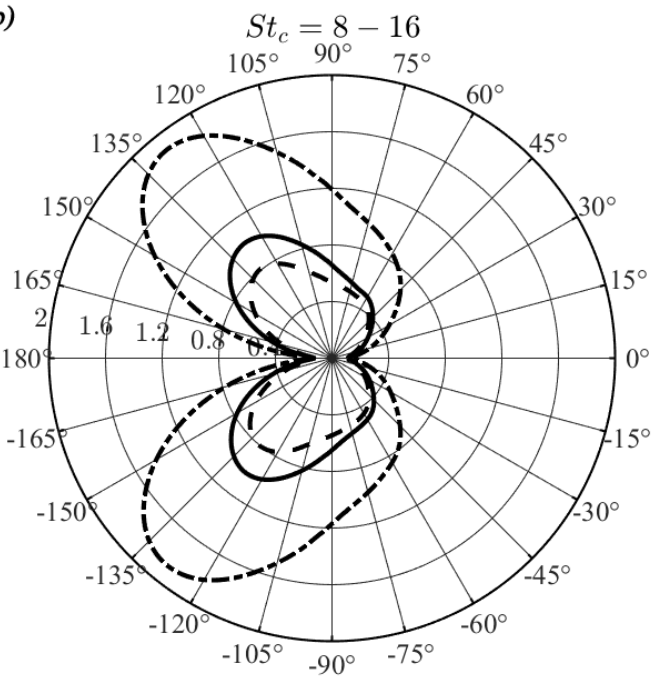

(c)

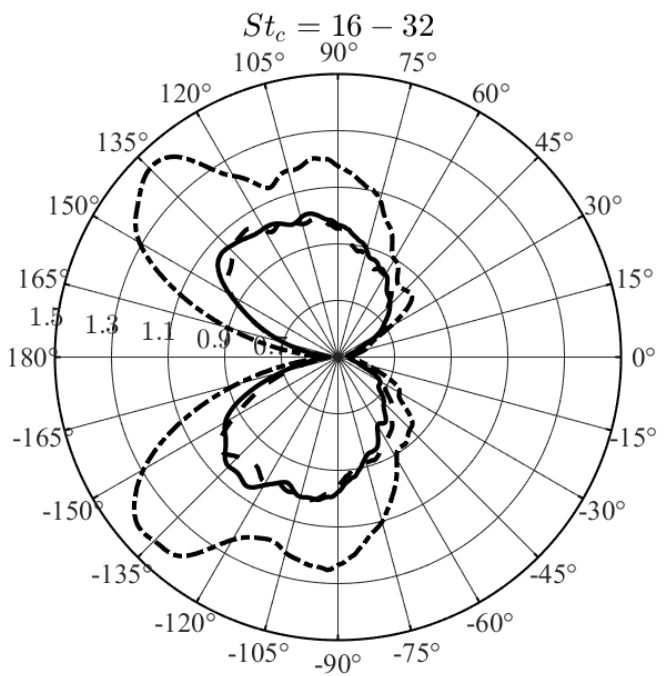

Fig. 5. Directivity plot for the straight, sawtooth and iron-shaped trailing-edge serrations. Data is obtained from microphones positioned in a circle of radius $10 c$ at the mid-span plane of the baseline trailing edge. Results are further averaged over the frequency band reported in each plot: (a): $2<S t_{c}<8$, (b) $8<S t_{c}<16$ and (c) $16<S t_{c}<32$.

The scaled acoustic power spectrum $\left(\Phi_{a a}\right)$ for the baseline airfoil and the ones with the sawtooth and iron-shaped trailing-edge serrations are plotted in Figure 4 (a). Spectra were evaluated by using Hanning windows of 500 elements and $50 \%$ of overlap, thus resulting in a frequency resolution of approximately $20 \mathrm{~Hz}$. Results in Figure 4 were further integrated over $1 / 10$ decade bandwidths. Spectra show broadband noise without a tonal component induced by the vortex shedding at the trailing-edge (see Section 3). Figure 4 (b) quantifies the noise reduction with respect to the baseline airfoil $\left(\Delta \Phi_{a a}\right)$. In the same figure, the far-field noise reduction measured by Arce León et al. [27] at $U_{\infty}=30 \mathrm{~m} / \mathrm{s}$ using the same sawtooth geometry is added for comparison. The measured noise reduction is comparable with the computed one. Focusing on the computation results, the iron-shaped geometry reduces the far-field 
broadband noise more than the conventional sawtooth geometry in the range of Strouhal number based on the chord $5<S t_{c}=f c / U_{\infty}<15$. Both geometries show maximum noise reduction at approximately $S t_{c}=10$. The maximum noise reduction is equal $8 \mathrm{~dB}$ for the iron geometry while it is equal to $6 \mathrm{~dB}$ for the sawtooth one. The comparison between the two serrated trailing-edges shows that the variation of the shape has a positive effect on the far-field noise, especially in the low frequency range.

To further compare the two serrated trailing-edges, directivity plots are shown in Figure 5. Data was obtained from microphones positioned in a circle of radius $10 c$ at the mid-span plane around the baseline trailing-edge. Results were further averaged over the frequency band reported in each plot. As expected, at low frequencies (Figure 5 a), a compact dipole source is seen at the trailing edge. Increasing the frequency (Figure $5 \mathrm{~b}$ ), the dipole is tilted toward the leading edge of the airfoil. Further increasing the frequency (Figure $5 \mathrm{c}$ ), a non-compact behavior appears only for the straight trailing-edge with different upstream oriented lobes. In this frequency range, no lobes are visible for the serrated cases. It is argued that this change is generated by the addition of the serrated geometry, thereby locally transferring non-compact sources back to compact ones. Noise reductions with respect to the baseline airfoil are observed at all angles with maximum for angles between 105 and 135 degrees. When comparing the two investigated serration geometries, it is evident that, at the highest simulated frequency, the two geometries generate the same noise intensity. It is also clear that the modification of the serration geometry does not alter the directivity pattern in the simulated frequency range.

\subsection{Mean and turbulent flow features}

In order to identify the physical mechanisms responsible for the larger noise reduction in presence of the iron-shaped serration, the mean and statistical flow fields are investigated. 

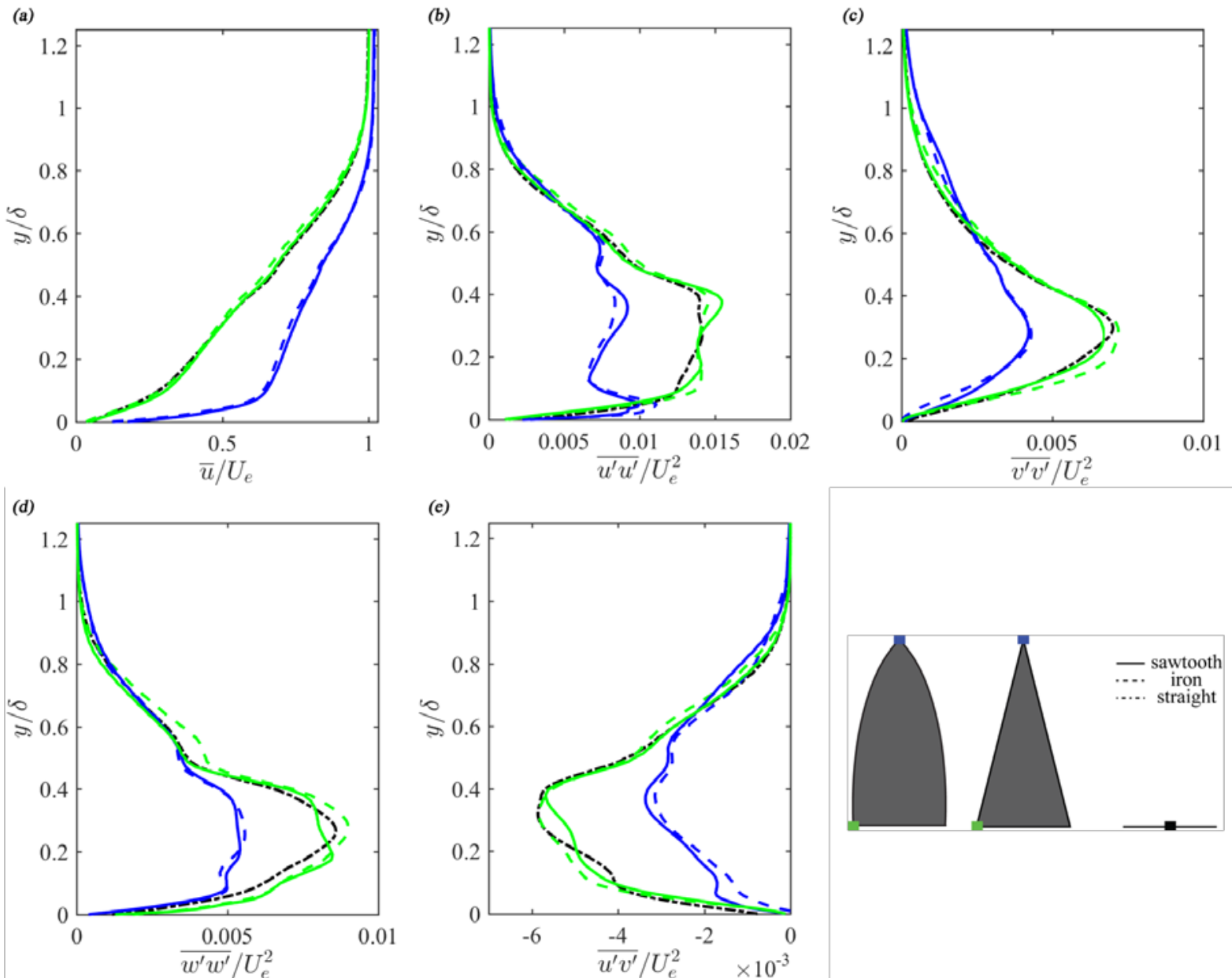

Fig. 6. Comparison of the time-averaged mean streamwise-velocity component (a) and of the time-averaged mean turbulent fluctuations (b-e) at $x / 2 h=0$ (blue line) and $x / 2 h=1$ (green line). Profiles of the baseline configuration are extracted at $z / c=0$ (black line).

Profiles of the time-averaged mean streamwise velocity component and of the time-averaged turbulent fluctuations are plotted in Figure 6. Wall-normal profiles at two points located at the root $(x / 2 h=0)$ and at the tip $(x / 2 h=1)$ of the investigated serrations are shown. The results obtained from the baseline configuration at the mid-span location $(z / c=0)$ are reported for the sake of completeness. 

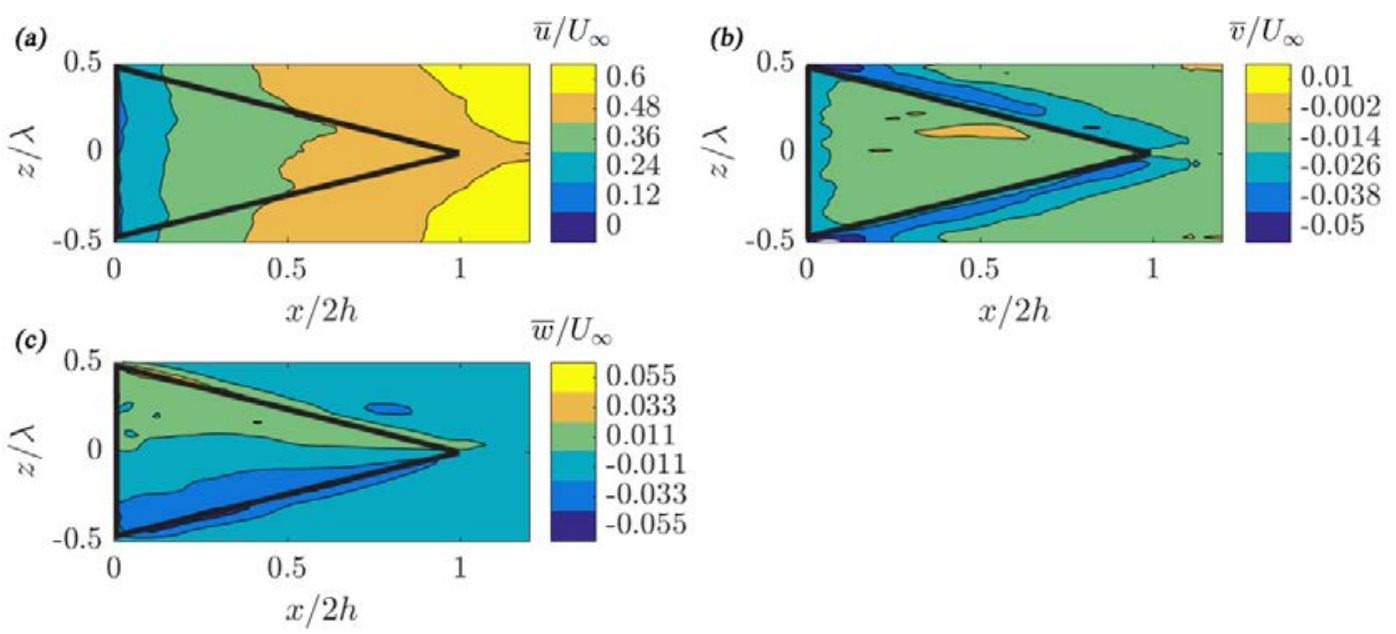

Fig. 7. Contour of the mean velocity component over the sawtooth serration at $y / \delta=0.05$ : (a) $\bar{u}$, (b) $\bar{v}$ and (c) $\bar{w}$ velocity components. Projections of the serration on the $x-z$ plane are indicated by means of continuous black lines.
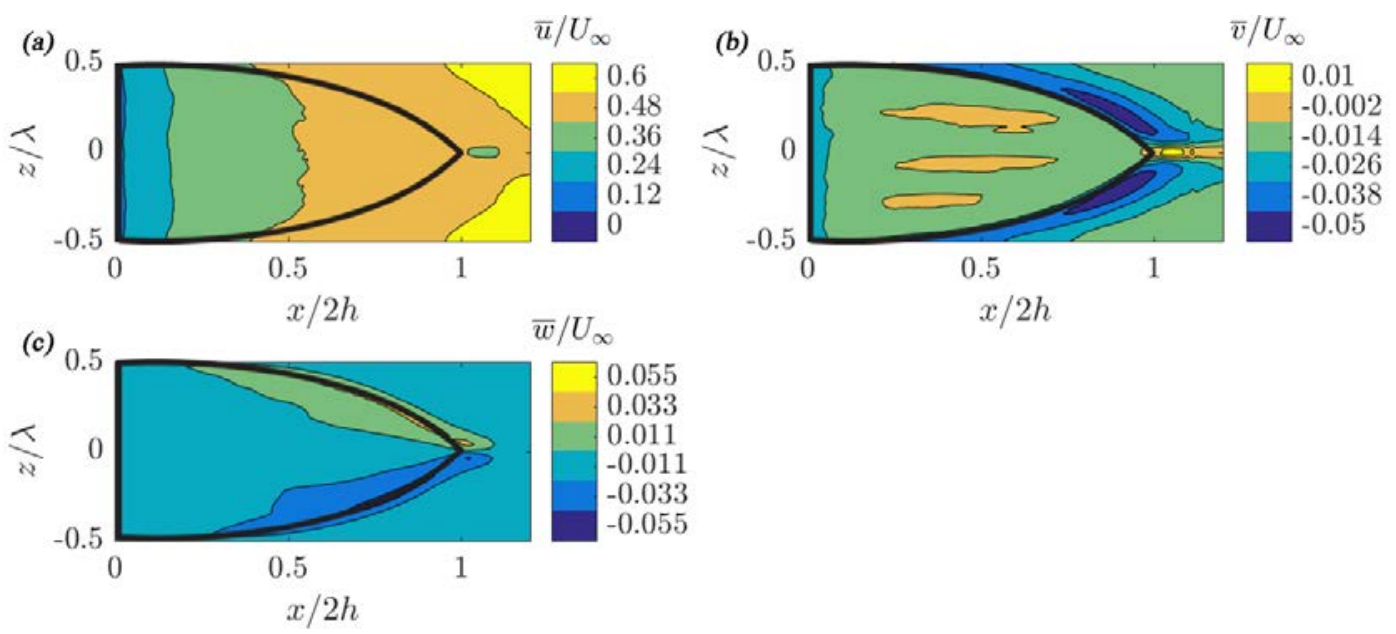

Fig. 8. Contour of the mean velocity component over the iron-shaped serration at $y / \delta=0.05$ : (a) $\bar{u}$, (b) $\bar{v}$ and (c) $\bar{w}$ velocity components. Projections of the serration on the $x-z$ plane are indicated by means of continuous black lines.

Data shows that the serrations mildly affect the boundary layer time-averaged mean and turbulent statistics at $x / 2 h=0$ with respect to the baseline airfoil. Similar results were reported in the experiments carried out by Chong \& Vathylakis [21] and Gruber et al. [19]. Strong similarities are present also at the tip $(x / 2 h=1)$. These results suggest that the turbulent flow convecting over the serration centerline is weakly sensitive to the serration geometry but it depends mainly on the streamwise location along the serration [10].

In presence of spanwise variable geometries, such as the conventional sawtooth geometry, the spanwise flow variation may form a three dimensional mixing layer consequently responsible for the streamwise oriented structures [50,51]. Previous studies $[10,24,25]$ showed that the sawtooth trailing-edge serrations induce distortion of the near-wall streamlines. Following Chong \& Vathylakis [21], the distortion of the streamlines may reduce the effective angle seen by the turbulent flow convecting over the serrations and thus may locally increase the scattered noise with respect to an ideal flow over a sawtooth geometry. 
In order to compare the two investigated serrations and inspect the physical reasons behind the computed far-field noise intensity, the near-wall spatial distributions of the time-averaged mean velocity components are discussed. They are reported in Figure 7 and Figure 8 for the conventional sawtooth and the iron-shaped geometry, respectively. Data is extracted at $y / \delta=0.05(y=0.5 \mathrm{~mm}$ above the serration). The mean streamwise velocity component increases from the root to the tip, corresponding to an acceleration of the flow with a thinning effect of the boundary layer. Most notably, the flow tends to seep into the empty space in between serrations (downward motion) as evidenced by the negative mean wall-normal velocity component $(\bar{v})$. As a direct consequence, the flow exhibits an outward motion as visible from the contour of the spanwise velocity component $(\bar{w})[7,10,22]$.

Similar flow features, but at a more downstream location $(x / 2 h>0.5)$, are present when retrofitting the airfoil with the iron-shaped serration. At the root, the reduced free space due the tangent constraint delays both the downward and the outward motions discussed above. More in detail, up to $x / 2 h=0.25$ the flow is characterized by $\bar{w}=0$. At approximately $x / 2 h=0.75$, both the upward and downward motions are enhanced and larger values of both $\bar{v}$ and $\bar{w}$ are measured. A second major difference between the two serrations is present at the tip. While the sawtooth serration is characterized by $\bar{v}$ approximately equal to zero (i.e., flow approximately parallel to the serration surface), the iron-shaped geometry is characterized by a strong upwash. It may be caused by the interaction between the vortical structures generated at the edge of the serration.

\subsection{Wall-pressure fluctuations}

The far field noise is generated by the scattering of turbulent flow convecting over the edge of the serrations. To further understand the reasons behind the lower far-field noise generated by the iron-shaped geometry, the time-averaged wall-pressure fluctuations $\left(\overline{p^{\prime} p^{\prime}}\right)$ are discussed (Figure 9). Both the spatial distribution and the intensity of the wall-pressure fluctuations do not depend on the serration geometry. The intensity of the time-averaged wall-pressure fluctuations is a function of the streamwise location while it is a weak function of the spanwise location. It decreases from the root to the tip suggesting variable intensity of the scattered pressure waves. In both cases, the intensity of $\overline{p^{\prime} p^{\prime}}$ is more than two times larger at the root than at the tip. It might be caused by the flow deviation imposed by the presence of the serration and the consequent variation of the pressure gradient. This assumption is supported by the mean wall-normal velocity component shown in Figure 7 and Figure 8, where $\bar{v}$ approximately equal to zero is measured for both configurations at $0<x / 2 h<0.1$.

The fact that $\overline{p^{\prime} p^{\prime}}$ do not depend on the serration geometry suggests that the lower far-field noise generated in presence of the iron-shaped geometry (Figure 4) is mainly due to the effective angle seen by the turbulent flow approaching the edge of the serrations and to the interference between scattered waves. To further confirm that the pressure fluctuations are only a function of the streamwise location, narrowband spectra of the wall-pressure fluctuations $\left(\Phi_{p p}\right)$ are plotted in Figure 10. Spectra were evaluated as described in Section 4.1. Three reference points along the edge of the serrations are taken at $x / 2 h=0,0.5$ and 1 . 

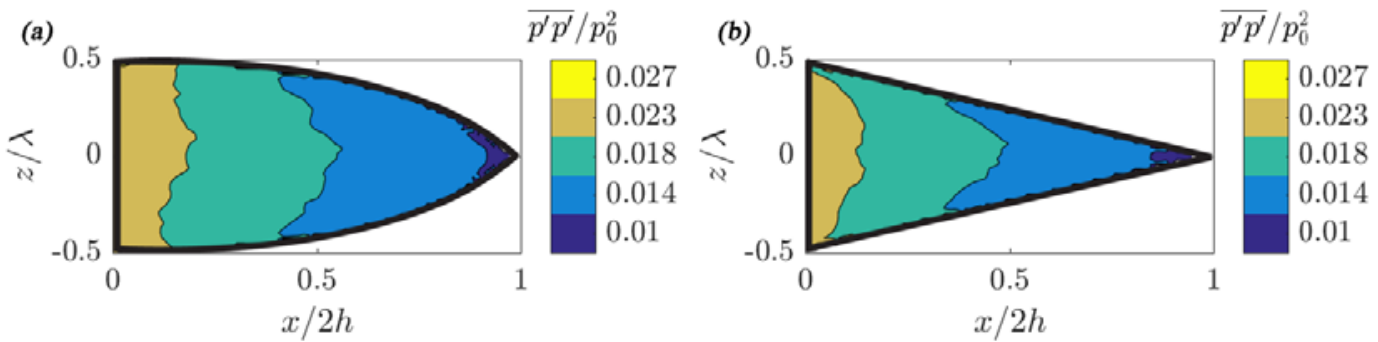

Fig. 9. Intensity of the mean wall-pressure fluctuations $\left(\overline{p^{\prime} p^{\prime}} / p_{0}^{2}\right)$ : (a) iron-shaped serration, (b) sawtooth serration. The serration on the $x-z$ plane are indicated by means of continuous black lines.

Spectra of the wall-pressure fluctuations show strong similarities between the two investigated geometries. In particular, no difference is measured at $x / 2 h=0.5$ and 1 , while minor differences are visible at $x / 2 h=0$. At this location, the intensity of $\Phi_{p p}$ is slightly larger (approximately $2 \mathrm{~dB}$ ) for the iron-shaped geometry in the low frequency range $\left(5<S t_{c}<7\right)$. Differently, at relatively higher frequencies $\left(S t_{c}>10\right)$ the iron-shaped geometry shows lower intensity of approximately $0.5 \mathrm{~dB}$.

A deeper analysis of Figure 10 suggests that far-field noise intensity benefits by both the reduced scattering efficiency due to the serration angle, and by the streamwise variation of $\Phi_{p p}$ with respect to the root location $\left(\Delta \Phi_{p p}=\Phi_{p p}^{\text {root }}-\Phi_{p p}^{\text {stream }}\right.$, where $\Phi_{p p}^{\text {root }}$ and $\Phi_{p p}^{\text {stream }}$ are the power spectra intensity at the root location and at the other streamwise points, respectively). As a matter of fact, in the frequency range where the $\Delta \Phi_{p p}$ between streamwise locations is larger (Figure 10), the estimated far-field noise reduction is higher.
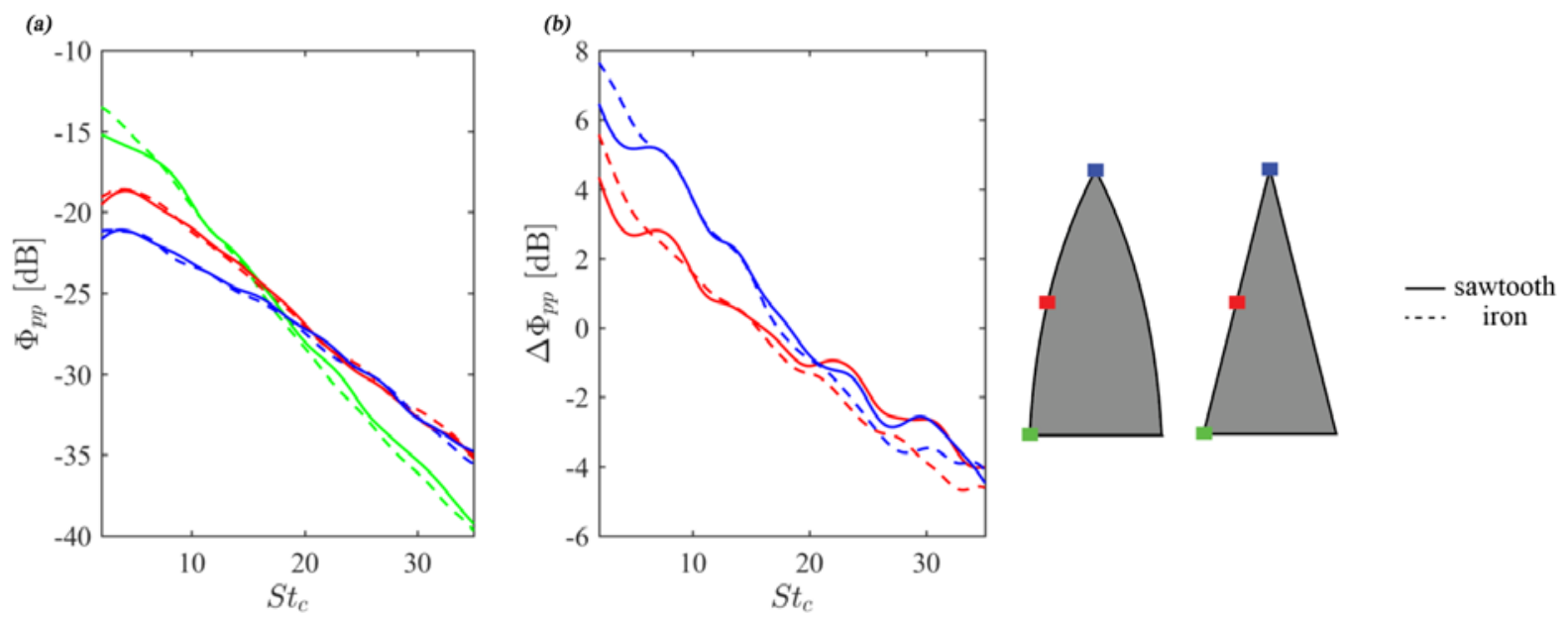

Fig. 10. (a) Spectra of the wall-pressure fluctuations $\left(\Phi_{p p}\right)$ at three streamwise locations corresponding to $x / 2 h=0$ (green), 0.5 (red), 1 (blue). The continuous and dashed lines represents the sawtooth and the iron-shaped serrations respectively. (b) $\Delta \Phi_{p p}=\Phi_{p p}^{\text {root }}-\Phi_{p p}^{\text {stream }}$, where $\Phi_{p p}^{\text {root }}$ and $\Phi_{p p}^{\text {stream }}$ are the power spectra intensity at the root location and at the other streamwise points, respectively.

The overall good agreement further confirms that the wall-pressure fluctuations are only dependent on the effective length of the serration and not on the actual shape. These findings support the assumption behind the shaped geometry as discussed in the introduction. The effect of the introduced curvature is to reduce the scattered noise at the trailing-edge root by 
mitigating both the interaction between the two sides of the airfoil and the negative effect induced by the outward and downward flow motions at this location (see Figures 7 and 8) [21]. The new geometry delays this effect downstream where the intensity of the pressure fluctuations is lower, thus contributing less to the far-field noise.

\section{Conclusions}

In this manuscript, computations of the flow convecting over a conventional sawtooth and an iron-shaped serration geometries were performed using the compressible, transient and explicit Lattice Boltzmann Method. The two investigated geometries had same length and wavelength and were retrofitted to a NACA 0018 airfoil. They were inspected in terms of both far-field noise and flow field.

The iron-shaped geometry reduces far-field noise more (approximately $2 \mathrm{~dB}$ ) than the conventional sawtooth geometry in the range $5<S t_{c}<15$, without modifying the directivity of the noise propagation in the similar frequency range. The analysis of the time-averaged near-wall velocity components shows that the main effect of the proposed geometry is to mitigate both the outward and downward flow motions near the root of the serration. It results in a milder interaction between the two sides of the airfoil at the root location, and, in a larger effective angle seen by the turbulent flow approaching the edges. On the other hand, stronger outward and downward flow motions are present near the serration tip. However, at this location, the intensity of wall-pressure fluctuations is lower, thus scattering less noise. In order to confirm that the achieved lower far-field noise is mainly due to the above mentioned flow effects, the time-averaged wall-pressure fluctuations and their spectra were inspected. It is found that they do not depend on the serration geometry, for a given serration aspect ratio, but that they are a function of the streamwise location. This finding confirms that the intensity of the scattered pressure waves depends on the streamwise location.

\section{Acknowledgments}

The research of the PhD candidate W.C.P. van der Velden is funded and supported by Siemens Wind Power A/S, Brande, Denmark.

\section{References}

[1] T.F. Brooks, D.S. Pope, M.A. Marcolini, Airfoil self-noise and prediction, National Aeronautics and Space Administration, Office of Management, Scientific and Technical Information Division, 1989.

[2] J.E.F. Williams, L.H. Hall, Aerodynamic sound generation by turbulent flow in the vicinity of a scattering half plane, J. Fluid Mech. 40 (1970) 657-670. doi:10.1017/S0022112070000368.

[3] S. Oerlemans, Reduction of wind turbine noise using blade trailing edge devices, in: 22nd AIAA/CEAS Aeroacoustics Conf., American Institute of Aeronautics and Astronautics, Lyon, France, France, 2016: pp. 1-18. doi:10.2514/6.2016-3018.

[4] S. Oerlemans, M. Fisher, T. Maeder, K. Kögler, Reduction of Wind Turbine Noise Using Optimized Airfoils and Trailing-Edge Serrations, AIAA J. 47 (2009) 1470-1481. http://arc.aiaa.org/doi/abs/10.2514/1.38888. 
[5] D.J. Moreau, C.J. Doolan, Noise-Reduction Mechanism of a Flat-Plate Serrated Trailing Edge, AIAA J. 51 (2013) 2513-2522. doi:10.2514/1.J052436.

[6] T.P. Chong, P.F. Joseph, M. Gruber, Airfoil self noise reduction by non-flat plate type trailing edge serrations, Appl. Acoust. 74 (2013) 607-613. doi:10.1016/j.apacoust.2012.11.003.

[7] C. Arce León, D. Ragni, S. Pröbsting, F. Scarano, J. Madsen, Flow topology and acoustic emissions of trailing edge serrations at incidence, Exp. Fluids. 57 (2016). doi:10.1007/s00348-016-2181-1.

[8] T. Dassen, R. Parchen, J. Bruggeman, F. Hagg, Results of a wind tunnel study on the reduction of airfoil self-noise by the application of serrated blade trailing edges, in: Proceeding Eur. Union Wind Energy Conf. Exhib., 1996: pp. 800-803.

[9] M. Gruber, P.F. Joseph, T.P. Chong, On the mechanisms of serrated airfoil trailing edge noise reduction, in: 17th AIAA/CEAS Aeroacoustics Conf., American Institute of Aeronautics and Astronautics, Portland, Oregon, Oregon, 2011: pp. 1-23. doi:10.2514/6.2011-2781.

[10] F. Avallone, S. Pröbsting, K.P. Lynch, D. Ragni, Tomographic-PIV investigation of the flow over serrated trailing-edges, in: 54th AIAA Aerosp. Sci. Meet. AIAA SciTech, American Institute of Aeronautics and Astronautics, San Diego, California, 2016: pp. 1-14. doi:10.2514/6.2016-1012.

[11] M. Gruber, Airfoil noise reduction by edge treatments, University of Southampton, 2012.

[12] C. Arce León, R. Merino-Martínez, D. Ragni, F. Avallone, M. Snellen, Boundary layer characterization and acoustic measurements of flow-aligned trailing edge serrations, Exp. Fluids. 57 (2016) 182. doi:10.1007/s00348-016-2272-z.

[13] M.S. Howe, Aerodynamic noise of a serrated trailing edge, J. Fluids Struct. 5 (1991) 33-45. doi:10.1016/0889-9746(91)80010-B.

[14] B. Lyu, M. Azarpeyvand, S. Sinayoko, Prediction of noise from serrated trailing edges, J. Fluid Mech. 793 (2016) 556-588. doi:10.1017/jfm.2016.132.

[15] M. Azarpeyvand, M. Gruber, P.F. Joseph, An analytical investigation of trailing edge noise reduction using novel serrations, in: 19th AIAA/CEAS Aeroacoustics Conf., American Institute of Aeronautics and Astronautics, Berlin, Germany, 2013: pp. 1-17. doi:10.2514/6.2013-2009.

[16] M.S. Howe, Noise produced by a sawtooth trailing edge, J. Acoust. Soc. Am. 90 (1991) 482-487. doi:10.1121/1.401273.

[17] B. Lyu, M. Azarpeyvand, S. Sinayoko, A Trailing-Edge Noise Model for Serrated Edges, in: 21st AIAA/CEAS Aeroacoustics Conf., American Institute of Aeronautics and Astronautics, Dallas, Texas, Texas, 2015: pp. 1-24. doi:10.2514/6.2015-2362.

[18] M. Drela, XFOIL: An Analysis and Design System for Low Reynolds Number 
Airfoils, in: Springer Berlin Heidelberg, 1989: pp. 1-12. doi:10.1007/978-3-64284010-4_1.

[19] M. Gruber, P.F. Joseph, T.P. Chong, Experimental investigation of airfoil self noise and turbulent wake reduction by the use of trailing edge serrations, in: 16th AIAA/CEAS Aeroacoustics Conf., American Institute of Aeronautics and Astronautics, Stockholm, Sweden, Sweden, 2010: pp. 1-23. doi:10.2514/6.2010-3803.

[20] R.K. Amiet, Noise due to turbulent flow past a trailing edge, J. Sound Vib. 47 (1976) 387-393. doi:10.1016/0022-460X(76)90948-2.

[21] T.P. Chong, A. Vathylakis, On the aeroacoustic and flow structures developed on a flat plate with a serrated sawtooth trailing edge, J. Sound Vib. 354 (2015) 65-90. doi:10.1016/j.jsv.2015.05.019.

[22] L.E. Jones, R.D. Sandberg, Acoustic and hydrodynamic analysis of the flow around an aerofoil with trailing-edge serrations, J. Fluid Mech. 706 (2012) 295-322. doi:10.1017/jfm.2012.254.

[23] H. Clemons, R.W. Wlezien, Modification of Flow Structures Associated with Broadband Trailing Edge Noise, in: 46th AIAA Fluid Dyn. Conf., American Institute of Aeronautics and Astronautics, Washington, D.C., D.C., 2016: pp. 1-18. doi:10.2514/6.2016-3627.

[24] F. Avallone, S. Pröbsting, D. Ragni, Three-dimensional flow field over a trailing-edge serration and implications on broadband noise, Phys. Fluids. 28 (2016) 117101. doi:10.1063/1.4966633.

[25] W.C.P. van der Velden, A. van Zuijlen, D. Ragni, Flow topology and noise emission around straight, serrated and slitted trailing edges using the Lattice Boltzmann methodology, in: 22nd AIAA/CEAS Aeroacoustics Conf., American Institute of Aeronautics and Astronautics, Reston, Virginia, 2016. doi:10.2514/6.2016-3021.

[26] M. Herr, W. Dobrzynski, Experimental Investigations in Low-Noise Trailing Edge Design, AIAA J. 43 (2005) 1167-1175. doi:10.2514/1.11101.

[27] C. Arce León, F. Avallone, S. Pröbsting, D. Ragni, PIV Investigation of the Flow Past Solid and Slitted Sawtooth Serrated Trailing Edges, in: 54th AIAA Aerosp. Sci. Meet. AIAA Scitech, American Institute of Aeronautics and Astronautics, San Diego, California, California, 2016: pp. 1-15. doi:10.2514/6.2016-1014.

[28] M. Gruber, P.F. Joseph, M. Azarpeyvand, An experimental investigation of novel trailing edge geometries on airfoil trailing edge noise reduction, in: 19th AIAA/CEAS Aeroacoustic Conf., American Institute of Aeronautics and Astronautics, Berlin, Germany, Germany, 2013: pp. 1-23. doi:10.2514/6.2013-2011.

[29] T.P. Chong, A. Vathylakis, P.F. Joseph, M. Gruber, Self-Noise Produced by an Airfoil with Nonflat Plate Trailing-Edge Serrations, AIAA J. 51 (2013) 2665-2677. doi:10.2514/1.J052344. 
[30] W.C.P. van der Velden, S. Oerlemans, Numerical analysis of noise reduction mechanisms on improved trailing edge serrations using the Lattice Boltzmann method, in: 35th Wind Energy Symp., American Institute of Aeronautics and Astronautics, Grapevine, Texas, 2017: pp. 1-24. doi:10.2514/6.2017-1379.

[31] P.M.H.W. Vijgen, F.G. Howard, D.M. Bushnell, B.J. Holmes, Serrated trailing edges for improving lift and drag characteristics of lifting surfaces, US 5088665 A, 1989.

[32] S. Oerlemans, Arrangement to reduce noise of a wind turbine rotor blade, EP2851553 A1, 2015.

[33] S. Succi, The lattice Boltzmann equation for fluid dynamics and beyond, 1st editio, Clarendon Press, Oxford, 2001.

[34] W.C.P. van der Velden, S. Pröbsting, A. van Zuijlen, A.T. de Jong, Y. Guan, S.C. Morris, Numerical and experimental investigation of a beveled trailing edge flow and noise field, J. Sound Vib. 384 (2016) 113-129. doi:10.1016/j.jsv.2016.08.005.

[35] P.L. Bhatnagar, E.P. Gross, M. Krook, A Model for Collision Processes in Gases. I. Small Amplitude Processes in Charged and Neutral One-Component Systems, Phys. Rev. 94 (1954) 511-525. doi:10.1103/PhysRev.94.511.

[36] H. Chen, S. Kandasamy, S. Orszag, R. Shock, S. Succi, V. Yakhot, Extended Boltzmann Kinetic Equation for Turbulent Flows, Science (80-. ). 301 (2003) 633-636. doi:10.1126/science.1085048.

[37] S. Chen, G.D. Doolen, LATTICE BOLTZMANN METHOD FOR FLUID FLOWS, Annu. Rev. Fluid Mech. 30 (1998) 329-364. doi:10.1146/annurev.fluid.30.1.329.

[38] K. Habibi, H. Gong, A. Najafiyazdi, L.G. Mongeau, Prediction of the Sound radiated from Low-Mach Internal Mixing Nozzles with Forced Mixers using the Lattice Boltzmann Method, in: 19th AIAA/CEAS Aeroacoustics Conf., Berlin, Germany, 2013: pp. 1-15. doi:10.2514/6.2013-2143.

[39] J.E.F. Williams, D.L. Hawkings, Sound Generation by Turbulence and Surfaces in Arbitrary Motion, Philos. Trans. R. Soc. London A Math. Phys. Eng. Sci. 264 (1969). doi:10.1098/rsta.1969.0031.

[40] F. Farassat, G.P. Succi, A review of propeller discrete frequency noise prediction technology with emphasis on two current methods for time domain calculations, J. Sound Vib. 71 (1980) 399-419. doi:10.1016/0022-460X(80)90422-8.

[41] G. Brès, F. Pérot, D. Freed, A Ffowcs Williams - Hawkings Solver for LatticeBoltzmann Based Computational Aeroacoustics, in: 16th AIAA/CEAS Aeroacoustics Conf., American Institute of Aeronautics and Astronautics, Reston, Virigina, 2010. doi:10.2514/6.2010-3711.

[42] D. Casalino, An advanced time approach for acoustic analogy predictions, J. Sound Vib. 261 (2003) 583-612. doi:10.1016/S0022-460X(02)00986-0.

[43] N. Curle, The Influence of Solid Boundaries upon Aerodynamic Sound, Proc. R. Soc. 
London A Math. Phys. Eng. Sci. 231 (1955) 505-514.

[44] A. Mann, M.-S. Kim, J. Wu, F. Perot, J. Grilliat, M.C. Jacob, M. Colman, Airfoil Tip Leakage Aeroacoustics Predictions using a Lattice Boltzmann Based Method, in: 22nd AIAA/CEAS Aeroacoustics Conf., American Institute of Aeronautics and Astronautics, Reston, Virginia, 2016. doi:10.2514/6.2016-2825.

[45] A. Powell, Theory of Vortex Sound, J. Acoust. Soc. Am. 36 (1964) 177. doi:10.1121/1.1918931.

[46] P.W. Bearman, Investigation of the flow behind a two-dimensional model with a blunt trailing edge and fitted with splitter plates, J. Fluid Mech. 21 (1965) 241. doi:10.1017/S0022112065000162.

[47] F. White, Viscous Fluid Flow, 3rd ed., McGraw-Hill Education, 2006.

[48] J.E.F. Williams, Hydrodynamic Noise, Annu. Rev. Fluid Mech. 1 (1969) 197-222. doi:10.1146/annurev.fl.01.010169.001213.

[49] W.K. Blake, Mechanics of Flow-Induced Sound and Vibration V2: Complex FlowStructure Interactions, Volume 2, Orlando, FL, Academic Press, Inc., 1986.

[50] I. Wygnanski, P. Tewes, H. Kurz, L. Taubert, C. Chen, The application of boundary layer independence principle to three-dimensional turbulent mixing layers, J. Fluid Mech. 675 (2011) 336-346. doi:10.1017/jfm.2011.95.

[51] R.W. Wlezien, V. Kibens, Passive control of jets with indeterminate origins, AIAA J. 24 (1986) 1263-1270. doi:10.2514/3.9430. 